\title{
Oncology bioanalysis: from biomarkers to drug discovery
}

\section{"The importance of well-validated and useful cancer biomarkers is especially acute in the setting of drug development."}

Keywords: biomarkers $\bullet$ cancer $\bullet$ diagnostic $\bullet$ therapies $\bullet$ validation

Despite impressive gains in the successful treatment of many cancers, mortality from cancer remains the second most common cause of death in the USA [1]. Globally, population demographics and shifting patterns of disease are predicted to result in an even greater increase in cancer incidence. It has been estimated that annual cancer cases will rise from 14 million in 2012 to 22 million within the next two decades [2]. Although there is a compelling need to introduce new therapies to address this problem, barriers to the development of novel oncology drugs remain daunting. Cancer is a heterogeneous disease, and cancer patients are a heterogeneous population with interindividual differences in the genetic basis of their cancers. Therefore, apart from a few marked successes with targeted therapies, the impact of personal medicine in cancer therapy has yet to be fully realized. The importance of wellvalidated and useful cancer biomarkers is especially acute in the setting of drug development. The availability of such biomarkers should lead to significant improvements in moving new therapeutics from bench to bedside by allowing researchers and clinicians to distinguish which patients will benefit from a specific therapy.

Impressive scientific and technological progress suggests that biomarkers could play an increasing role in improving oncology drug discovery. Specifically, robust analytical methods including mass spectrometry and various forms of nuclear magnetic resonance spectroscopy now provide detailed quantitative information on patients' responses to therapy. Important contributions have been made to diagnostic and prognostic biomarkers which are a prerequisite for identifying patients who will benefit from therapy. The fundamental understanding of drug absorption, distribution, metabolism and excretion in oncology are constantly improving. In the areas of imaging analysis, drug localization and cancer metabolism, ongoing studies have revealed new approaches [3], improved targeted therapies [4] and revealed new metabolic pathways [5].

The articles in this themed issue review recent advances in critical areas of oncology bioanalysis. Biomarker discovery, validation, translation and clinical applications are highlighted. The cutting-edge tools and studies used to make these advances are also discussed together with the applicability of biomarkers in different stages of cancer from cancer risk assessment to evaluating recurrence. It is clear that considerable challenges remain for bioanalytical scientists including improving existing biomarkers, adapting biomarkers to new biologic therapeutics, validating biomarkers and translating the validated biomarkers to oncology drug development and the clinic.

Financial \& competing interests disclosure

The authors have no relevant affiliations or financial involvement with any organization or entity with a financial interest in or financial conflict with the subject matter or materials discussed in the manuscript. This includes employment, consultancies, honoraria, stock ownership or options, expert testimony, grants or patents received or pending, or royalties.

No writing assistance was utilized in the production of this manuscript.
Biomarkers

in Medicine
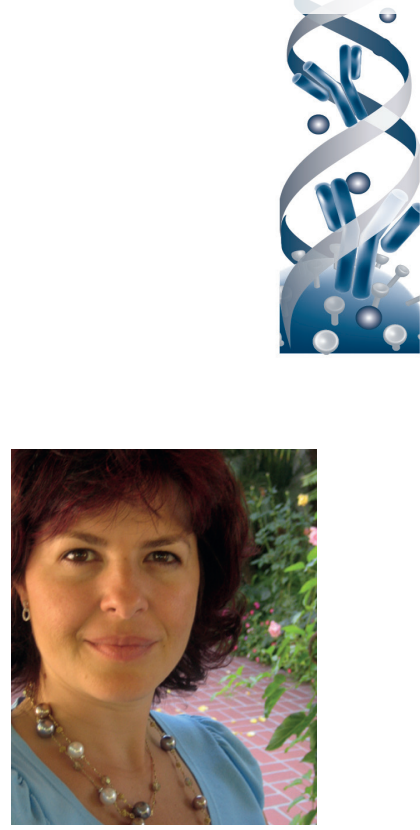

Clementina Mesaros

Penn SRP Center, Center of Excellence in Environmental Toxicology, Department of Systems Pharmacology \& Translational Therapeutics, Perelman School of Medicine, University of Pennsylvania, Philadelphia, PA 19104-6160, USA

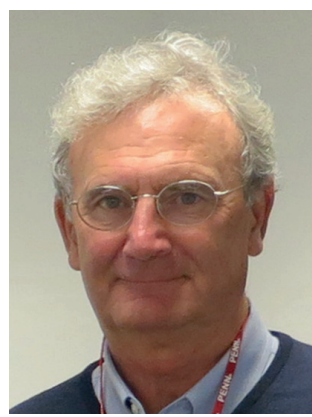

Ian A Blair

Author for correspondence: Penn SRP Center, Center of Excellence in Environmental Toxicology, Department of Systems Pharmacology \& Translational Therapeutics, Perelman School of Medicine, University of Pennsylvania, Philadelphia, PA 19104-6160, USA and

Center for Cancer Pharmacology, University of Pennsylvania, Rm 854 BRB II/III, 421 Curie Blvd, Philadelphia, PA 19104, USA

Tel.: +1 2155739885

Fax: +1 2155739889

ianblair@mail.med.upenn.edu

\section{Future $\because \cdots$ Medicine $\%$}




\section{References}

1 Cancer Facts \& Figures 2014. www.cancer.org

2 WHO, Media centre. www.who.int/mediacentre/factsheets/fs297/en

3 Veselkov KA, Mirnezami R, Strittmatter N et al. Chemo-informatic strategy for imaging mass spectrometry-based hyperspectral profiling of lipid signatures in colorectal cancer. Proc. Natl Acad. Sci. USA 111(3), 1216-1221 (2014).

4 Sapra P, Betts A, Boni J. Preclinical and clinical pharmacokinetic/pharmacodynamic considerations for antibody-drug conjugates. Expert Rev. Clin. Pharmacol. 6(5), 541-555 (2013).

5 Ward PS, Lu C, Cross JR et al. The potential fo isocitrate dehydrogenase mutations to produce 2-hydroxyglutarate depends on allele specificity and subcellular compartmentalization. J. Biol. Chem. 288(6), 3804-3815 (2013).

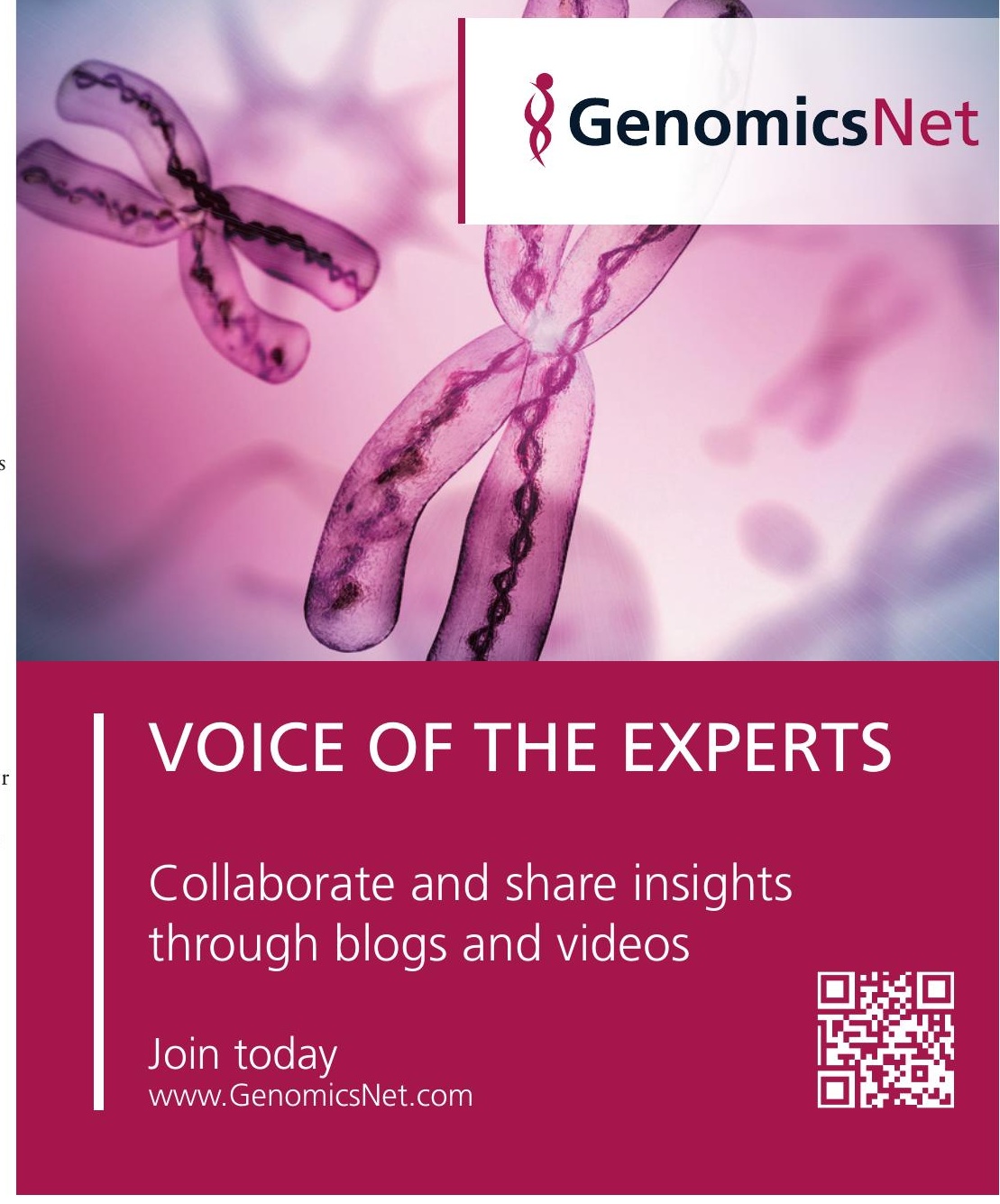

